\title{
The methods of using the sword of Shinkage-ryu-kenjutsu and the Contemporary Kendo: The techniques of the body for manipulating a bamboo sword
}

\author{
Koya SATO ${ }^{*}$, Fumiaki SHISHIDA 2 , \& Teruo OBOKI ${ }^{3}$ \\ ${ }^{1}$ Graduate School of Sport Sciences, Waseda University (Japan) \\ ${ }^{2}$ Faculty of Sport Sciences, Waseda University (Japan) \\ ${ }^{3}$ Emeritus Professor, Saitama University (Japan)
}

\section{5th IMACSSS World Scientific Congress Abstracts, Rio Maior (Portugal), October 6-8 \\ Section: Historical, sociological and philosophical issues of MA\&CS \\ Type: Oral communication}

\section{Introduction}

Nowadays the Japan Kendo Federation guides practitioners to develop mind and body through competing of Yuko-datotsu while they practice kendo. Yuko-datotsu is defined in the Match and judge rules. The main contents are as follows: "showing the fullness of spirit", "appropriate posture", "striking the zone of an opponent that are permitted by striking the part from tip of the Shinai (the bamboo sword) to around 30 centimeters, while imaging to cut along Hasuji (a line that connects a back and edge of Japanese sword)", and "expressing Zanshin (Preparation for a counterattack)".

To cut along Hasuji means to manipulate the Shinai as Nihonto (Japanese sword). Therefore, this author thinks that for the improvement of the quality of modern kendo, it is important to reexamine how to manipulate the Shinai along Hasuji.

The purpose of this study is to clarify techniques of the body for manipulating a Shinai through examining Toho (the methods of using the sword) in Shinkage-ryu kenjutsu, which was founded by Kamiizumi Hidetsuna, and systematized in the sixteenth century, the Edo era. The concept of Toho that was defined by Takeharu Kiyono(1927-2008), who did a deep research about Onoha-itto-ryukenjutsu. Onoha-itto-ryu-kenjutsu comprises the manipulation of Mogi-to (imitation-sword) such as a Fukuro-shinai (bamboo sword wrapped by leather). Toho of Shinkage-ryu has been handed down up to now. One of characteristics of Shinkage-ryu is to use the Fukuro-shinai. It is an alternative of Japanese sword to strike an opponent safely and forcefully. Thus, this study examines how to manipulate Fukuro-shinai from the following two viewpoints.

1. From the viewpoints of the relation between a practitioner and Fukuro-shinai.

2. When and how to manipulate the Fukuro-shinai against opponents?

\section{Methodology}

This is a historical study combined with fieldwork. By field work we gathered the information about Toho of Shinkage-ryu which is not sufficiently recorded yet and the information is related to core techniques and the training system materials. We also carried out interviews set at a place closed to leaders of the dojo of Shinkage-ryu in Chiba prefecture. The field work also contains participant observation (PO) about practices in the dojo, which is intended to obtain inside information. 


\section{Results}

(1) From the viewpoints of the relation between a practitioner and Fukuro-shinai.

First, acquire Go-ka-no-narai (the five important teachings) in manipulating a Fukuro-shinai. Go-kano-narai means: 1) To hold your body so as to present only one side to your opponent; 2) To hold your shoulder to be on a plane with your opponent's; 3) To make a shield of your own fists; 4) To extend your left arm; 5) To rest weight on a forward knee and stretch out a rear leg. Go-ka-no-narai reside in all techniques of katas in Shinkage-ryu.

(2) How to manipulate a Fukuro-shinai against an opponent, and how to time.

1) A Shidachi (winner in a kata) should give attention to a moment that an opponent try to strike by a bamboo sword.

2) A Shidachi and an Uchitachi (looser in a kata) should give attention to a moment that they stroke each other with using a Fukuro-shinai.

3) Practitioners should manipulate a Fukuro-shinai to cut along Hasujie ach moment.

\section{Discussion and conclusion}

Regarding the first perspective in the result, we are convinced that Go-ka-no-narai are basic lessons that always manifest themselves when practicing each techniques of all katas in Shinkage-ryu.

Regarding the second perspective in the results, it is considered that 1) a reason giving attention to a moment is to control Uchitachi's attack while manipulating a Fukuro-shinai to cut along Hasuj; 2) there are three types of conditions manipulating right Hasuji as follow: A. To make a body turn by centering on the backbone; B. To extend left arm; C. To grip tight a Fukuro-shinai as if wring out a towel; 3) there are three types of conditions controlling Uchitachi's attack as follow: A. To catch the moment when Uchitachi tries to attack; B. To step forward earlier than Uchitachi; C. To advance towards the center of an opponent who moves.

The techniques of the body performing along Hasuji is to integrate the movement of a body, hands, feet, and Mogi-to such as Shinai or Fukuro-shinai into one. The acquisition of such basics and methods are determined by workmanship of Jumonji-kachi (the core technique of Shinkage-ryu), and a practitioner can gradually acquire such basics and methods by learning Shinkage-ryu training system.

In conclusion, there are techniques of the body that guarantee correct Hasuji in Shinkage-ryu even in case that a tool such as Fukuro-shinai changes Shinai in the modern kendo. Hence it is consider that practitioners can practice contemporary kendo with performing to cut along Hasuji by Fukuroshinai.

Key words: Kendo; Kenjutsu; Hasuji; Fukuro-shinai; Go-ka-no-narai; Jumonji-kachi.

This study was supported in part by Grants-in-Aid for Scientific Research from the Japan Society for the Promotion of Science (Fundamental Research (B), Task No. 15H03067, Study representative: Fumiaki Shishida). 